\title{
Two cases of clozapine induced thrombocytosis
}

\author{
N Liyanage, N Gunarathna, J Mendis
}

Abstract

Cytopaenias are known to be associated with clozapine treatment. Thrombocytosis maybe mediated by immune reaction and can be of use as potential markers for clozapine induced agranulocytosis, in the future. There are only few case reports of clozapine induced thrombocytosis in the literature. This article discusses two case reports of secondary thrombocytosis associated with clozapine.

SL J Psychiatry 2016; 7(1): 26-27

\section{Introduction}

Blood dyscrasias associated with clozapine include leucopenia, neutropenia and thrombocytopenia (1-3\% of patients) and anaemia, leucocytosis and thrombocytosis ( $<1 \%$ of patients) (1). Some of these abnormalities may be due to the direct action of clozapine on the haematopoietic stem cells of the bone marrow, mediated through immune reactions which can create a picture similar to myeloid leukaemia or myelopro-liferative disorder. Therefore abnormalities of haematocrit or platelets, including thrombocytosis may be of use as a potential marker for future clozapine induced severe agranulocytosis (1).

\section{Case report 1}

A 45-year old single female with a long history of schizophrenia had been started on clozapine 5 years previously due to poor response to other treatment. Her symptoms had improved with clozapine. However, she had discontinued treatment against medical advice. Thereafter she was treated with monthly intramuscular flupenthixol and oral antipsychotic medications. Her medication compliance had been poor and she was admitted to the hospital with suspiciousness, irritability and assaultive behaviour. On admission she was noted to have persecutory and bizarre delusions. She continued to have symptoms in hospital while on multiple medications and it was decided to re-start her on clozapine. Her pre-clozapine blood investigations revealed an anaemia (Hb-10.2g/dl) with normal WBC $\left(5400 / \mathrm{mm}^{3}\right)$ and platelet $\left(271,000 / \mathrm{mm}^{3}\right)$ counts. The full blood count was monitored weekly while gradually titrating up the dose of clozapine.

Eighteen days after starting clozapine treatment, at a dose of 550mg daily, she developed a lower respiratory tract infection (LRTI) with fever. Investigations done at the time showed a platelet count of $437,000 / \mathrm{mm}^{3}$ and WCC of $10,300 / \mathrm{mm}^{3}$. The clozapine dose was reduced to $150 \mathrm{mg}$ daily and she was treated with intravenous co-amoxiclav and oral clarithromycin, and the LRTI resolved within 3 days. The clozapine was again titrated to $250 \mathrm{mg}$ daily on the $22^{\text {nd }}$ day of therapy. The third weekly blood test revealed evidence of thrombocytosis, with a platelet count of $764,000 / \mathrm{mm}^{3}$. The blood picture at that time showed an increased number of platelets with normal morphology and concomitant eosinophilia. She did not have any features suggestive of myocarditis. Given these changes, the clozapine was omitted after $27^{\text {th }}$ day of commencement and the platelet count returned to normal $\left(318,000 / \mathrm{mm}^{3}\right)$ after stopping clozapine. Clozapine was not started again and she was managed with high doses of other antipsychotic medications, and her platelet count remained within normal limits.

\section{Case 2}

A 33-year old male patient with a history of schizophrenia since the age of 19 was admitted to a surgical ward following a motor vehicle accident, due to a non-displaced fracture of the right humerus, which was managed by immobilisation. He did not sustain head injuries. On admission he was muttering to himself and had delusions of persecution and poor self-care. He had no significant medical problems. Except for chewing betel, he did not use substances. He had been started on clozapine during his last psychiatric admission 8 months previously, after failing to respond to several antipsychotic medications. At that time he had been discharged on clozapine 200mg daily. He had become non-complaint with medication soon after the discharge and was lost to follow-up. He had not been on psychotropic medication for the last 7 months and had continued to have psychotic symptoms including suspiciousness, muttering to self suggestive of hallucinatory experiences, and occasional irritability. After discussion with the patient and family, he was restarted on clozapine $12.5 \mathrm{mg}$ daily, 10 days after the admission. Investigations done immediately prior to commencement of clozapine were normal and the platelet count was in upper normal range $\left(377 \times 10^{3}\right)$. His clozapine dose was titrated up to $150 \mathrm{mg}$ daily in divided doses over the next 7 days. The first weekly blood 
investigations after clozapine re-commencement revealed an elevated platelet count $\left(501 \times 10^{3}\right)$ without any abnormality in other blood indices. He complained of excessive drowsiness and hyper-salivation, which were attributed to clozapine. He had no other physical symptoms. His psychotic symptoms, with the exception of poor self-care, showed moderate improvement. His clozapine dose was reduced to $100 \mathrm{mg}$ due to the reported side effects. The patient complained of non-specific generalised body pain in the next few days and his platelet count remained elevated on day 11 of clozapine treatment $\left(496 \times 10^{3}\right)$. Other blood indices including ESR and CRP were within normal range. The platelet count became normal $\left(360 \times 10^{3}\right)$ on day 13 . His nonspecific body pain had improved by that time. His platelet count remained within normal range during his hospital stay.

\section{Discussion}

Platelets, an acute-phase reactant, may increase in response to various stimuli, including anaemia, systemic infections, inflammatory conditions, haemorrhage or malignancy; this phenomenon is known as secondary thrombocytosis (2).

In our first patient, the thrombocytosis may have been secondary to persistent anaemia, the lower respiratory tract infection or treatment with clozapine. This patient had low haemoglobin (Hb-10.2g/dl) on admission to the hospital, even though her baseline platelet count was normal. The platelet count remained normal up until $23^{\text {rd }}$ day of starting clozapine, while she had a low haemoglobin throughout this period. Therefore thrombocytosis is unlikely to be due to co existent anaemia. However, further investigations are needed to rule out this possibility.

Thrombocytosis secondary to infection is well known (2). This patient had features of a lower respiratory tract infection by the 18th day of commencement of clozapine. Although at the time of infection she had a leucocytosis, her platelet count was in the normal range for 6 days after the onset of infection. Therefore thrombocytosis is unlikely to be due to lower respiratory tract infection. In this patient thrombocytosis occurred while re-titrating with clozapine and the platelet count returned back to normal after clozapine was stopped. Therefore in this case thrombocytosis was most probably due to clozapine treatment, however thrombocytosis secondary to infection cannot be ruled out completely.

While our $2^{\text {nd }}$ patient had experienced recent trauma, the observed thrombocytosis is unlikely to be due to the trauma, given the time which had elapsed between the onset of thrombocytosis and the trauma. Our $2^{\text {nd }}$ patient developed thrombocytosis soon after re-introduction of clozapine. Hampson reported a case of thrombocytosis soon after reintroduction of clozapine (3). Eranti and Chaturvedi reported thrombocytosis preceded by thrombocytopenia 28-30 weeks into clozapine therapy (4). Hampson and Marlowe identified thrombocytosis or thrombocytopaenia associated with clozapine as part of an immune reaction and the case reported by Hampson developed arthralgia, malaise, sweating and nausea along with thrombocytocis $(1,3)$. However Eranti and Chaturvedi did not observe signs of inflammation in their patient. While our patient had mild manifestations suggestive of systemic involvement, he did not have an elevated ESR as in the case reported by Hampson.

Both Hampson, Eranti and Chathurvedi reported a return of platelet count to normal levels, within a short period of time without specific intervention as seen in our patient. Some authors speculated possible association of platelet count with clozapine dose and we noticed reduction of platelet count with reduction of dose of clozapine, although this may have been purely incidental (4).

\section{Conclusions}

There are only few case reports of clozapine induced thrombocytosis in the literature. Both patients described in this case series developed secondary thrombocytosis associated with clozapine. Considering that they did not have manifestation suggestive of other stimuli, the observed increase in platelet count is most likely due to clozapine - namely, a clozapine induced thrombocytosis. While it is reported that thrombocytosis may predict agranulocytosis, this was not seen in our patients.

\section{Conflicts of interest}

None declared

N Liyanage, N Gunarathna, J Mendis, National Institute of Mental Health, Angoda

Corresponding author: $\mathrm{N}$ Liyanage

Email: ulnsliyanage@gmail.com

\section{References}

1. Marlowe K. Thrombocytosis due to clozapine treatment: working towards an early marker for clozapine-induced agranulocytosis. Br J Psychiatry 2000; 177: 372-3.

2. Vora AJ, Lilleyman JS. Secondary thrombocytosis. Arch Dis Child 1993; 68(1): 88-90.

3. Hampson ME. Clozapine-induced thrombocytosis. Br J Psychiatry 2000; 176: 400.

4. Savithasri Eranti V, Chaturvedi SK. Marked thrombocyte count variations without agranulocytosis due to clozapine. Indian J Psychiatry 1998; 40(3): 300-2. 